\title{
Fluoro, Alkysulfanyl, and Alkylsulfonyl Leaving Groups in Suzuki Cross-Coupling Reactions of Purine 2'-Deoxynucleosides and Nucleosides
}

\author{
Jiangqiong Liu and Morris J. Robins* \\ Department of Chemistry and Biochemistry, Brigham Young University, \\ Provo, Utah-84602-5700 \\ Morris_robins@byu.edu
}

Supporting Information 1

Pages S2-S7: Experimental procedures and characterization data 


\section{EXPERIMENTAL DETAILS AND CHARACTERIZATION DATA}

Spectral Methods: ${ }^{1} \mathrm{H}(500 \mathrm{MHz})$ and ${ }^{13} \mathrm{C}(125 \mathrm{MHz})$ spectra were determined with solutions in $\mathrm{CDCl}_{3}$. High-resolution mass spectra (HRMS) were determined with FAB (glycerol, $\mathrm{NaOAc})$ unless otherwise indicated.

2',3',5'-Tri- $\boldsymbol{O}$-(2,4,6-trimethylbenzoyl)adenosine. Adenosine (1.44 g, $5.38 \mathrm{mmol})$ was added to a cold $\left(0{ }^{\circ} \mathrm{C}\right)$ sitrred solution of 2,4,6-trimethylbenzoic acid (4.00 g, $\left.24.4 \mathrm{mmol}\right)$ and trifluoroacetic anhydride $(3.45 \mathrm{~mL}, 5.12 \mathrm{~g}, 24.4 \mathrm{mmol})$ in $\mathrm{CH}_{2} \mathrm{Cl}_{2}(200 \mathrm{~mL})$, and stirring was continued for $3 \mathrm{~h}$. The reaction mixture was poured into saturated $\mathrm{NaHCO}_{3} / \mathrm{H}_{2} \mathrm{O}$, and the organic phase was washed (brine) and dried $\left(\mathrm{Na}_{2} \mathrm{SO}_{4}\right)$. Volatiles were evaporated, and the residue was chromatographed $\left(\mathrm{CH}_{2} \mathrm{Cl}_{2} / \mathrm{MeOH} 50: 1\right)$ and recrystallized from EtOH to give the title compound (3.41 g, 89\%): $\mathrm{mp} 208-210{ }^{\circ} \mathrm{C} ;{ }^{1} \mathrm{H}$ NMR $\delta 2.05,2.18,2.29(3 \times \mathrm{s}, 3 \times 6 \mathrm{H}), 2.24,2.28,2.30(3 \times$ $\mathrm{s}, 3 \times 3 \mathrm{H}), 4.71(\mathrm{dd}, J=8.3,4.4 \mathrm{~Hz}, 1 \mathrm{H}), 4.84(\mathrm{dd}, J=12.2,3.4 \mathrm{~Hz}, 1 \mathrm{H}), 4.92(\mathrm{dd}, J=12.2,4.9$ $\mathrm{Hz}, 1 \mathrm{H}), 5.77$ (br s, 2H), 6.11 (t, $J=4.9 \mathrm{~Hz}, 1 \mathrm{H}), 6.32$ (d, J=5.4 Hz, 1H), 6.45 (t, J=5.3 Hz, 1H), 6.77, 6.82, $6.87(3 \times \mathrm{s}, 3 \times 2 \mathrm{H}), 7.89,8.32(2 \times \mathrm{s}, 2 \times 1 \mathrm{H}) ;{ }^{13} \mathrm{C}$ NMR $\delta 20.10,20.12,21.37$, 21.39, 21.45, 63.9, 71.8, 73.7, 80.9, 86.8, 120.3, 128.80, 128.83, 128.9, 129.4, 130.2, 135.5, $135.9,136.2,139.3,140.0,140.3,140.5,150.1,153.7,155.7,168.5,168.9,169.8 ;$ HRMS $m / z$ $728.3055\left[\mathrm{MNa}^{+}\left(\mathrm{C}_{40} \mathrm{H}_{43} \mathrm{~N}_{5} \mathrm{O}_{7} \mathrm{Na}\right)=728.3060\right]$.

\section{6-Fluoro-9-[2,3,5-tri-O-(2,4,6-trimethylbenzoyl)- $\beta$-D-ribofuranosyl]purine. General}

Method 1. 2',3',5'-Tri- $O$-(2,4,6-trimethylbenzoyl)adenosine (1.25 g, $1.77 \mathrm{mmol})$ was added to a solution of $\mathrm{HF} /$ pyridine $(55 \%, 10 \mathrm{~mL})$ at $-15^{\circ} \mathrm{C}$, and tert-butyl nitrite (TBN) $(2.5 \mathrm{~mL}, 2.2 \mathrm{~g}, 21$ mmol) was added dropwise. The solution was stirred at $-15^{\circ} \mathrm{C}$ for $15 \mathrm{~min}$, and cold $\mathrm{H}_{2} \mathrm{O}$ and $\mathrm{CH}_{2} \mathrm{Cl}_{2}$ were added. The organic layer was washed $\left(\mathrm{NaHCO}_{3} / \mathrm{H}_{2} \mathrm{O}\right.$, brine $)$ and dried $\left(\mathrm{Na}_{2} \mathrm{SO}_{4}\right)$. Volatiles were evaporated, and the residue was chromatographed (EtOAc/hexanes, 3:7). The purified material was recrystallized from EtOH to give the title compound (1.02 g, 81\%): $\mathrm{mp}$ 117-120 ${ }^{\circ} \mathrm{C} ;{ }^{1} \mathrm{H}$ NMR $\delta 2.07,2.19(2 \times \mathrm{s}, 2 \times 6 \mathrm{H}), 2.25(\mathrm{~s}, 9 \mathrm{H}), 2.28,2.32(2 \times \mathrm{s}, 2 \times 3 \mathrm{H})$, 4.71-4.81 (m, 3H), 6.12-6.15 (m, 1H), 6.37-6.40 (m, 2H), 6.78, 6.83, $6.87(3 \times \mathrm{s}, 3 \times 2 \mathrm{H}), 8.18$, $8.55(2 \times \mathrm{s}, 2 \times 1 \mathrm{H}) ;{ }^{13} \mathrm{C}$ NMR $\delta 20.0,20.08,20.09,21.3,21,4,63.4,71.5,73.9,81.1,87.6$, $121.1\left(\mathrm{~d}, J_{\mathrm{C}, \mathrm{F}}=29 \mathrm{~Hz}\right), 128.6,128.81,128.86,128.92,129.2,129.9,135.4,135.9,136.1,140.2$, 140.4, 143.8, $152.5\left(\mathrm{~d}, J_{\mathrm{C}, \mathrm{F}}=14 \mathrm{~Hz}\right), 155.0\left(\mathrm{~d}, J_{\mathrm{C}, \mathrm{F}}=11 \mathrm{~Hz}\right), 160.0\left(\mathrm{~d}, J_{\mathrm{C}, \mathrm{F}}=261 \mathrm{~Hz}\right), 168.5$ 168.8, 169.7; HRMS $m / z 731.2856\left[\mathrm{MNa}^{+}\left(\mathrm{C}_{40} \mathrm{H}_{41} \mathrm{~N}_{4} \mathrm{FO}_{7} \mathrm{Na}\right)=731.2857\right]$. 


\section{6-Phenyl-9-[2,3,5-tri- $O$-(2,4,6-trimethylbenzoyl)- $\beta$-D-ribofuranosyl]purine (1a).}

Treatment of 6-fluoro-9-[2,3,5-tri-O-(2,4,6-trimethylbenzoyl)- $\beta$-D-ribofuranosyl]purine (30 mg, $0.042 \mathrm{mmol}$ ) and phenylboronic acid by general method 2 gave $1 \mathrm{a}$ (30 $\mathrm{mg}, 84 \%)$ as a solid glass: ${ }^{1} \mathrm{H}$ NMR $\delta$ 2.07, 2.20, $2.26(3 \times \mathrm{s}, 3 \times 6 \mathrm{H}), 2.25,2.27,2.29(3 \times \mathrm{s}, 3 \times 3 \mathrm{H}), 4.73-4.84(\mathrm{~m}, 3 \mathrm{H})$, $6.18(\mathrm{t}, J=5.0 \mathrm{~Hz}, 1 \mathrm{H}), 6.41(\mathrm{~d}, J=5.0 \mathrm{~Hz}, 1 \mathrm{H}), 6.48(\mathrm{~d}, J=5.5 \mathrm{~Hz}, 1 \mathrm{H}), 6.77(\mathrm{~s}, 2 \mathrm{H}), 6.83(\mathrm{~s}$, 4H), 7.55-7.61 (m, 3H), 8.76-8.78 (m, 2H), 8.21, 8.96 (2 × s, $2 \times 1 \mathrm{H}) ;{ }^{13} \mathrm{C}$ NMR $\delta 20.1,21.4$, 63.7, 71.7, 73.7, 81.0, 87.2, 128.81, 128.85, 128.89, 128.93, 129.4, 130.1, 131.4, 131.8, 135.5, $135.7,135.9,136.1,140.0,140.4,140.5,143.1,152.2$, 152.9, 155.6, 168.6, 168.9, 169.8; HRMS $m / z 789.3273\left[\mathrm{MNa}^{+}\left(\mathrm{C}_{46} \mathrm{H}_{46} \mathrm{~N}_{4} \mathrm{O}_{7} \mathrm{Na}\right)=789.3264\right]$.

\section{6-(4-Methylphenyl)-9-[2,3,5-tri- $O$-(2,4,6-trimethylbenzoyl)- $\beta$-D-}

ribofuranosyl]purine (1b). Treatment of 6-fluoro-9-[2,3,5-tri- $O-(2,4,6$-trimethylbenzoyl)- $\beta$-Dribofuranosyl]purine $(30 \mathrm{mg}, 0.042 \mathrm{mmol})$ and 4-methylphenylboronic acid by general method 2 gave $1 \mathbf{b}(27 \mathrm{mg}, 82 \%)$ as a solid glass: ${ }^{1} \mathrm{H}$ NMR $\delta 2.06,2.20,2.25(3 \times \mathrm{s}, 3 \times 6 \mathrm{H}), 2.24,2.27$, 2.28, $2.47(4 \times \mathrm{s}, 4 \times 3 \mathrm{H}), 4.72-4.83(\mathrm{~m}, 3 \mathrm{H}), 6.17(\mathrm{t}, J=5.4 \mathrm{~Hz}, 1 \mathrm{H}), 6.41(\mathrm{~d}, J=4.9 \mathrm{~Hz}, 1 \mathrm{H})$, $6.46(\mathrm{t}, J=5.3 \mathrm{~Hz}, 1 \mathrm{H}), 6.77(\mathrm{~s}, 2 \mathrm{H}), 6.83(\mathrm{~s}, 4 \mathrm{H}), 7.39(\mathrm{~d}, J=8.0 \mathrm{~Hz}, 2 \mathrm{H}), 8.68$ (d, $J=8.0 \mathrm{~Hz}$,

2H), 8.19, $8.93(2 \times \mathrm{s}, 2 \times 1 \mathrm{H}) ;{ }^{13} \mathrm{C}$ NMR $\delta 20.08,20.11,21.4,21.9,63.7,71.7,73.7,81.0,87.1$, $128.80,128.85,128.9,129.4,129.7,130.0,131.6,132.9,135.5,135.9,136.1,140.0,140.4$, $140.5,141.9,142.8,152.1,152.9,155.6,168.6,168.9,169.8$; HRMS $m / z 803.3428\left[\mathrm{MNa}^{+}\right.$ $\left.\left(\mathrm{C}_{47} \mathrm{H}_{48} \mathrm{~N}_{4} \mathrm{O}_{7} \mathrm{Na}\right)=803.3421\right]$.

\section{6-(4-Methoxyphenyl)-9-[2,3,5-tri- $O$-(2,4,6-trimethylbenzoyl)- $\beta$-D-}

ribofuranosyl]purine (1c). General Method 2. (Procedure A). Under a flushing atmosphere of argon in a glove bag, 6-fluoro-9-[2,3,5-tri-O-(2,4,6-trimethylbenzoyl)- $\beta$-D-ribofuranosyl]purine (50 mg, $0.070 \mathrm{mmol}), \mathrm{Ni}(\mathrm{COD})_{2}(2 \mathrm{mg}, 0.007 \mathrm{mmol} ; 0.1$ equiv.), $\mathrm{IPr} \bullet \mathrm{HCl}(3.0 \mathrm{mg}, 0.007 \mathrm{mmol}$; 0.1 equiv.), 4-methoxyphenylboronic acid (21.3 mg, 0.14 mmol; 2 equiv.), and $\mathrm{K}_{3} \mathrm{PO}_{4}$ (45 mg, $0.21 \mathrm{mmol} ; 3$ equiv.) were added to a Schlenk flask containing a magnetic stir bar. The flask was evacuated and refilled with $\operatorname{Ar}(3 \times)$. The flask was then charged with dried THF, and the mixture was heated at $60{ }^{\circ} \mathrm{C}$ with stirring for $3 \mathrm{~h}$. The mixture was allowed to cool to ambient temperature, filtered, and the filter cake was washed with EtOAc. Volatiles were removed in vacuo, and the residue was chromatographed (EtOAc/hexanes, 1:4) to give 1c (47 mg, 84\%) as a solid glass: ${ }^{1} \mathrm{H}$ NMR $\delta$ 2.06, 2.20, $2.26(3 \times \mathrm{s}, 3 \times 6 \mathrm{H}), 2.24,2.27,2.28(3 \times \mathrm{s}, 3 \times 3 \mathrm{H}), 3.92(\mathrm{~s}$, 
$3 \mathrm{H}), 4.73-4.84(\mathrm{~m}, 3 \mathrm{H}), 6.17(\mathrm{t}, J=5.0 \mathrm{~Hz}, 1 \mathrm{H}), 6.41(\mathrm{~d}, J=5.4 \mathrm{~Hz}, 1 \mathrm{H}), 6.46(\mathrm{t}, J=5.4 \mathrm{~Hz}$, 1H), 6.77 (s, 2H), 6.83 (s, 4H), 7.09 (d, $J=9.0 \mathrm{~Hz}, 2 \mathrm{H}), 8.80(\mathrm{~d}, J=9.5 \mathrm{~Hz}, 2 \mathrm{H}), 8.18,8.90(2 \times$ $\mathrm{s}, 2 \times 1 \mathrm{H}) ;{ }^{13} \mathrm{C}$ NMR $\delta 20.1,21.4,55.6,63.7,71.7,73.7,81.0,87.0,114.3,128.4,128.8,128.9$, $129.4,130.1,131.2,131.9,135.5,135.9,136.2,140.0,140.3,140.5,142.5,152.0,152.9,155.2$, 162.4, 168.5, 168.9, 169.8; HRMS $m / z$ 819.3361 [ $\left.\mathrm{MNa}^{+}\left(\mathrm{C}_{47} \mathrm{H}_{48} \mathrm{~N}_{4} \mathrm{O}_{8} \mathrm{Na}\right)=819.3370\right]$.

6-(4-Fluorophenyl)-9-[2,3,5-tri- $O$-(2,4,6-trimethylbenzoyl)- $\beta$-D-ribofuranosyl]purine (1d). Treatment of 6-fluoro-9-[2,3,5-tri- $O$-(2,4,6-trimethylbenzoyl)- $\beta$-D-ribofuranosyl]purine (30 $\mathrm{mg}, 0.042 \mathrm{mmol}$ ) and 4-fluorophenylboronic acid by general method 2 gave $1 \mathrm{~d}$ (24 $\mathrm{mg}$, $73 \%)$ as a solid glass: ${ }^{1} \mathrm{H}$ NMR $\delta 2.07,2.19,2.25(3 \times \mathrm{s}, 3 \times 6 \mathrm{H}), 2.25,2.27,2.28(3 \times \mathrm{s}, 3 \times 3 \mathrm{H})$, 4.73-4.84 (m, 3H), $6.17(\mathrm{t}, J=5.0 \mathrm{~Hz}, 1 \mathrm{H}), 6.41(\mathrm{~d}, J=5.0 \mathrm{~Hz}, 1 \mathrm{H}), 6.46(\mathrm{t}, J=5.5 \mathrm{~Hz}, 1 \mathrm{H})$, $6.77(\mathrm{~s}, 2 \mathrm{H}), 6.83(\mathrm{~s}, 4 \mathrm{H}), 7.24-7.28(\mathrm{~m}, 2 \mathrm{H}), 8.83-8.86(\mathrm{~m}, 2 \mathrm{H}), 8.20,8.92(2 \times \mathrm{s}, 2 \times 1 \mathrm{H}) ;{ }^{13} \mathrm{C}$ NMR $\delta 20.1,21.4,63.7,71.6,73.7,81.0,87.2,116.0\left(\mathrm{~d}, J_{\mathrm{C}, \mathrm{F}}=21 \mathrm{~Hz}\right), 128.8,128.86,128.90$, $129.3,130.0,131.5,131.9,132.3\left(\mathrm{~d}, J_{\mathrm{C}, \mathrm{F}}=9 \mathrm{~Hz}\right), 135.5,135.9,136.1,140.0,140.4,140.6$, 143.1, 152.2, 152.9, 154.3, $165.0\left(\mathrm{~d}, J_{\mathrm{C}, \mathrm{F}}=251 \mathrm{~Hz}\right), 168.6,168.9,169.8 ; \mathrm{HRMS} \mathrm{m} / z$ 807.3185 $\left[\mathrm{MNa}^{+}\left(\mathrm{C}_{46} \mathrm{H}_{45} \mathrm{~N}_{4} \mathrm{O}_{7} \mathrm{Na}\right)=807.3170\right]$.

9-[2-Deoxy-3,5-di-O-(4-methylbenzoyl)- $\beta$-D-erythro-pentofuranosyl]-6-fluoropurine. Treatment of 2'-deoxy-3',5'-di-O-(4-methylbenzoyl)adenosine ${ }^{1}(0.50 \mathrm{~g}, 1.0 \mathrm{mmol})$ by general method 1 gave the title compound $(0.36 \mathrm{~g}, 71 \%)$ as solid foam after chromatography: ${ }^{1} \mathrm{H}$ NMR $\delta$ 2.39, $2.43(2 \times \mathrm{s}, 2 \times 3 \mathrm{H}), 2.87-2.91(\mathrm{~m}, 1 \mathrm{H}), 3.15-3.20(\mathrm{~m}, 1 \mathrm{H}), 4.64-4.68(\mathrm{~m}, 2 \mathrm{H}), 4.78(\mathrm{dd}, J$ $=13.7,5.4 \mathrm{~Hz}, 1 \mathrm{H}), 5.83-5.85(\mathrm{~m}, 1 \mathrm{H}), 6.59(\mathrm{dd}, J=7.8,5.9 \mathrm{~Hz}, 1 \mathrm{H}), 7.20(\mathrm{~d}, J=8.0 \mathrm{~Hz}, 2 \mathrm{H})$, $7.27(\mathrm{~d}, J=8.5 \mathrm{~Hz}, 2 \mathrm{H}), 7.86(\mathrm{~d}, J=8.5 \mathrm{~Hz}, 2 \mathrm{H}), 7.96(\mathrm{~d}, J=7.5 \mathrm{~Hz}, 2 \mathrm{H}), 8.28,8.56(2 \times \mathrm{s}, 2 \times$ $1 \mathrm{H}) ;{ }^{13} \mathrm{C}$ NMR $\delta 21.9,22.0,38.2,64.0,75.2,83.6,85.7,121.2\left(\mathrm{~d}, J_{\mathrm{C}, \mathrm{F}}=29 \mathrm{~Hz}\right), 126.5,126.8$, $129.5,129.6,129.8,130.0,143.5,144.5,144.8,152.2\left(\mathrm{~d}, J_{\mathrm{C}, \mathrm{F}}=14 \mathrm{~Hz}\right), 155.0\left(\mathrm{~d}, J_{\mathrm{C}, \mathrm{F}}=10 \mathrm{~Hz}\right)$, $160.0\left(\mathrm{~d}, J_{\mathrm{C}, \mathrm{F}}=260 \mathrm{~Hz}\right), 166.1,166.3$; HRMS $m / z 513.1560\left[\mathrm{MNa}^{+}\left(\mathrm{C}_{26} \mathrm{H}_{23} \mathrm{~N}_{4} \mathrm{FO}_{5} \mathrm{Na}\right)=\right.$ $513.1550]$.

\section{9-[2-Deoxy-3,5-di-O-(4-methylbenzoyl)- $\beta$-D-erythro-pentofuranosyl]-6-(4-}

methylphenyl)purine $^{2}$ (1e). Treatment of 9-[2-deoxy-3,5-di-O-(4-methylbenzoyl)- $\beta$-D-erythropentofuranosyl]-6-fluoropurine (50 $\mathrm{mg}, 0.10 \mathrm{mmol})$ and 4-methylphenylboronic acid by general method 2 gave 1e (34 mg, 60\%) as a solid glass with ${ }^{1} \mathrm{H}$ and ${ }^{13} \mathrm{C}$ NMR spectra identical to those of an authentic sample ${ }^{2}$ of $\mathbf{1 e .}$ 


\section{9-[2-Deoxy-3,5-di-O-(4-methylbenzoyl)- $\beta$-D-erythro-pentofuranosyl]-6-(4-}

fluorophenyl)purine ${ }^{3}$ (1f). Treatment of 9-[2-deoxy-3,5-di-O-(4-methylbenzoyl)- $\beta$-D-erythropentofuranosyl]-6-fluoropurine $(50 \mathrm{mg}, 0.10 \mathrm{mmol})$ and 4-fluorophenylboronic acid by general method 2 gave 1f (39 mg, 67\%) as a solid glass with ${ }^{1} \mathrm{H}$ and ${ }^{13} \mathrm{C}$ NMR spectral data the same as reported $^{3}$ for $\mathbf{1 f}$.

\section{9-(2,3,5-Tri- $O$-acetyl- $\beta$-D-ribofuranosyl)-6-[(3-methylbutyl)sulfanyl]purine.}

Treatment of 6-chloro-9-(2,3,5-tri- $O$-acetyl- $\beta$-D-ribofuranosyl)purine (1.0 g, $2.4 \mathrm{mmol})$ by general method 3 gave the title compound $(0.87 \mathrm{~g}, 75 \%)$ as a solid glass: ${ }^{1} \mathrm{H}$ NMR $\delta$ 0.95, $0.97(2$ $\times \mathrm{s}, 2 \times 3 \mathrm{H}), 1.64-1.68(\mathrm{~m}, 2 \mathrm{H}), 1.79(\mathrm{sept}, J=6.8 \mathrm{~Hz}, 1 \mathrm{H}), 2.07,2.12,2.14(3 \times \mathrm{s}, 3 \times 3 \mathrm{H})$, $3.39(\mathrm{t}, J=7.3 \mathrm{~Hz}, 2 \mathrm{H}), 4.36(\mathrm{dd}, J=12.9,5.0 \mathrm{~Hz}, 1 \mathrm{H}), 4.43-4.45(\mathrm{~m}, 2 \mathrm{H}), 5.66(\mathrm{t}, J=4.9 \mathrm{~Hz}$, $1 \mathrm{H}), 5.95(\mathrm{t}, J=5.4 \mathrm{~Hz}, 1 \mathrm{H}), 6.20(\mathrm{~d}, J=4.9 \mathrm{~Hz}, 1 \mathrm{H}), 8.11,8.70(2 \times \mathrm{s}, 2 \times 1 \mathrm{H}) ;{ }^{13} \mathrm{C} \mathrm{NMR} \delta$ 20.6, 20.8, 21.0, 22.5, 27.2, 27.7, 38.4, 63.2, 70.8, 73.3, 80.6, 86.6, 132.1, 141.1, 148.1, 152.3, 162.5, 169.5, 169.8, 170.5; HRMS $m / z 481.1743\left[\mathrm{MH}^{+}\left(\mathrm{C}_{21} \mathrm{H}_{29} \mathrm{~N}_{4} \mathrm{O}_{7} \mathrm{~S}\right)=481.1757\right]$.

\section{9-[2,3,5-Tri-O-(4-methylbenzoyl)- $\beta$-D-ribofuranosyl]-6-[(3-}

methylbutyl)sulfanyl]purine. General Method 3. 3-Methyl-1-butanethiol (0.38 mL, $320 \mathrm{mg}$, $3.0 \mathrm{mmol})$ was added to a solution of 6-chloro-9-[2,3,5-tri- $O$-(4-methylbenzoyl)- $\beta$-Dribofuranosyl]purine $^{4}(500 \mathrm{mg}, 0.761 \mathrm{mmol})$ and DBU $(0.14 \mathrm{~mL}, 140 \mathrm{mg}, 0.94 \mathrm{mmol})$ in MeCN $(4 \mathrm{~mL})$ at ambient temperature. The solution was stirred until the displacement was complete ( $\sim 30 \mathrm{~min}$, TLC). Cold $0.01 \mathrm{M} \mathrm{HCl} / \mathrm{H}_{2} \mathrm{O}$ was added, and the solution was extracted with $\mathrm{CH}_{2} \mathrm{Cl}_{2}$ $(50 \mathrm{~mL})$. The organic layer was washed $\left(\mathrm{NaHCO}_{3} / \mathrm{H}_{2} \mathrm{O}\right.$, brine $)$ and dried $\left(\mathrm{Na}_{2} \mathrm{SO}_{4}\right)$. Volatiles were evaporated, and the residue was chromatographed (EtOAc/hexanes, 3:7) to give the title compound (440 mg, 82\%) as a solid foam: ${ }^{1} \mathrm{H}$ NMR $\delta 0.96,0.97(2 \times \mathrm{s}, 2 \times 3 \mathrm{H}), 1.64-1.68(\mathrm{~m}$, 2H), 1.78 (sept, $J=6.8 \mathrm{~Hz}, 1 \mathrm{H}), 2.38(\mathrm{~s}, 3 \mathrm{H}), 2.42$ (s, 6H), 3.37-3.40 (m, 2H), 4.67 (dd, $J=$ 12.2, $4.4 \mathrm{~Hz}, 1 \mathrm{H}), 4.80-4.82(\mathrm{~m}, 1 \mathrm{H}), 4.88(\mathrm{dd}, J=12.0,3.4 \mathrm{~Hz}, 1 \mathrm{H}), 6.20(\mathrm{dd}, J=5.6,4.8 \mathrm{~Hz}$, $1 \mathrm{H}), 6.38(\mathrm{t}, J=5.8 \mathrm{~Hz}, 1 \mathrm{H}), 6.45(\mathrm{~d}, J=5.8 \mathrm{~Hz}, 1 \mathrm{H}), 7.16-7.25(\mathrm{~m}, 6 \mathrm{H}), 7.81-8.00(\mathrm{~m}, 6 \mathrm{H})$, 8.10, 8.64 $(2 \times \mathrm{s}, 2 \times 1 \mathrm{H}) ;{ }^{13} \mathrm{C}$ NMR $\delta 21.9,22.5,27.1,27.7,38.3,63.7,71.7,73.9,81.2,86.9$, $125.9,126.2$, 126.8, 129.4, 129.5, 129.6, 130.0, 130.09, 130.11, 132.1, 141.3, 144.4, 144.8, 148.3, 152.4, 162.4, 165.3, 165.6, 166.4; HRMS $m / z 735.2517\left[\mathrm{MNa}^{+}\left(\mathrm{C}_{39} \mathrm{H}_{40} \mathrm{~N}_{4} \mathrm{O}_{7} \mathrm{SNa}\right)=\right.$ 731.2515]. This material was used for coupling reactions without further purification.

9-[2,3,5-tri- $O$-(2,4,6-trimethylbenzoyl)- $\beta$-D-ribofuranosyl]-6-[(3methylbutyl)sulfanyl]purine. Treatment of 6-chloro-9-[2,3,5-tri-O-(2,4,6-trimethylbenzoyl)- $\beta$ - 
D-ribofuranosyl]purine ${ }^{4}(500 \mathrm{mg}, 1.45 \mathrm{mmol})$ by general method 3 gave the title compound (460 $\mathrm{mg}, 85 \%)$ as a solid foam: ${ }^{1} \mathrm{H}$ NMR $\delta 0.98(\mathrm{~d}, J=6.4 \mathrm{~Hz}, 6 \mathrm{H}), 1.66-1.70(\mathrm{~m}, 2 \mathrm{H}), 1.81$ (sept, $J$ $=6.8 \mathrm{~Hz}, 1 \mathrm{H}), 2.05,2.18,2.26(3 \times \mathrm{s}, 3 \times 6 \mathrm{H}), 2.24,2.28,2.30(3 \times \mathrm{s}, 3 \times 3 \mathrm{H}), 3.39-3.42(\mathrm{~m}$, 2H), 4.70-4.83 (m, 3H), $6.11(\mathrm{t}, J=4.9 \mathrm{~Hz}, 1 \mathrm{H}), 6.33(\mathrm{~d}, J=5.4 \mathrm{~Hz}, 1 \mathrm{H}), 6.37$ (t, $J=5.4 \mathrm{~Hz}$, $1 \mathrm{H}), 6.76,6.82,6.85(3 \times \mathrm{s}, 3 \times 2 \mathrm{H}), 8.01,8.63(2 \times \mathrm{s}, 2 \times 1 \mathrm{H}) ;{ }^{13} \mathrm{C}$ NMR $\delta$ 20.1, 20.2, 21.38, $21.4,22.6,27.2,27.8,38.4,63.8,71.7,73.7,81.0,87.0,128.7,128.81,128.84,128.9,129.4$, 130.1, 132.1, 135.5, 135.9, 136.2, 140.0, 140.4, 140.5, 141.3, 148.2, 152.5, 162.4, 168.5, 168.8, 169.8; HRMS $m / z$ 815.3441 [ $\left.\mathrm{MNa}^{+}\left(\mathrm{C}_{45} \mathrm{H}_{52} \mathrm{~N}_{4} \mathrm{O}_{7} \mathrm{SNa}\right)=815.3454\right]$.

\section{9-[2,3,5-Tri-O-(4-methylbenzoyl)- $\beta$-D-ribofuranosyl]-6-(4-methylphenyl)purine ${ }^{2}$}

(3a). Treatment of 9-[2,3,5-tri- $O$-(4-methylbenzoyl)- $\beta$-D-ribofuranosyl]-6-[(3-

methylbutyl)sulfanyl]purine ( $50 \mathrm{mg}, 0.07 \mathrm{mmol}$ ) and 4-methylphenylboronic acid by general method 4 gave 3a (34 mg, 69\%) as a solid glass with ${ }^{1} \mathrm{H}$ and ${ }^{13} \mathrm{C}$ NMR spectra identical to those of an authentic sample ${ }^{2}$ of $\mathbf{3 a}$.

\section{9-(2,3,5-Tri-O-acetyl- $\beta$-D-ribofuranosyl)-6-(4-methoxyphenyl)purine ${ }^{5}$ (3b). General}

Method 4. 9-(2,3,5-Tri- $O$-acetyl- $\beta$-D-ribofuranosyl)-6-[(3-methylbutyl)sulfanyl]purine (50 mg, $0.10 \mathrm{mmol}), \mathrm{Pd}(\mathrm{OAc})_{2}(2.4 \mathrm{mg}, 0.011 \mathrm{mmol} ; 0.11$ equiv.), $\mathrm{IPr} \bullet \mathrm{HCl}(4.5 \mathrm{mg}, 0.011 \mathrm{mmol} ; 0.11$ equiv.), 4-methoxyphenylboronic acid (30.4 mg, $0.20 \mathrm{mmol} ; 2.0$ equiv.), and $\mathrm{K}_{2} \mathrm{CO}_{3}$ (43 mg, $0.31 \mathrm{mmol} ; 3.1$ equiv.) were added to a Schlenk flask containing a magnetic stir bar. The flask was evacuated and refilled with argon ( $3 x)$. The flask was then charged with dried toluene, and the mixture was heated at $90{ }^{\circ} \mathrm{C}$ with stirring for $8 \mathrm{~h}$. The mixture was allowed to cool to ambient temperature, filtered, and the filter cake was washed with EtOAc. Volatiles were removed in vacuo, and the residue was chromatographed (EtOAc/hexanes 1:4) to give $\mathbf{3 b}(39 \mathrm{mg}, 78 \%$ ) as a solid glass: ${ }^{1} \mathrm{H}$ NMR spectral data were the same as reported ${ }^{5}$ and ${ }^{13} \mathrm{C}$ NMR $\delta$ 20.6, 20.7, 55.6, 63.3, 70.9, 73.3, 80.6, 86.5, 114.3, 128.3, 131.3, 131.8, 142.1, 152.1, 152.9, 155.3, 162.5, 169.5, 169.7, 170.5; HRMS $m / z$ 507.1484 $\left[\mathrm{MNa}^{+}\left(\mathrm{C}_{23} \mathrm{H}_{24} \mathrm{~N}_{4} \mathrm{O}_{8} \mathrm{Na}\right)=507.1492\right]$.

6-(4-Fluorophenyl)-9-[2,3,5-tri-O-(4-methylbenzoyl)- $\beta$-D-ribofuranosyl]purine ${ }^{2}$ (3c). Treatment of 9-[2,3,5-tri-O-(4-methylbenzoyl)- $\beta$-D-ribofuranosyl]-6-[(3methylbutyl)sulfanyl]purine (50 $\mathrm{mg}, 0.07 \mathrm{mmol}$ ) and 4-fluorophenylboronic acid by general method 4 gave $3 \mathbf{c}$ (35 mg, 71\%) as a solid glass with ${ }^{1} \mathrm{H}$ and ${ }^{13} \mathrm{C}$ NMR spectra identical to those of an authentic sample ${ }^{2}$ of $\mathbf{3 c}$. 


\section{9-[2,3,5-Tri-O-(2,4,6-trimethylbenzoyl)- $\beta$-D-ribofuranosyl]-6-[(3-}

methylbutyl)sulfonyl]purine (4). A solution of Oxone (780 mg, $1.26 \mathrm{mmol})$ in $\mathrm{NaOAc} / \mathrm{HOAc}$ $(1 \mathrm{M}, 30 \mathrm{~mL})$ buffer was added dropwise to a vigorously stirred solution of 9-[2,3,5-tri-O-(2,4,6trimethylbenzoyl)- $\beta$-D-ribofuranosyl]-6-[(3-methylbutyl)sulfanyl]purine $(0.50 \mathrm{~g}, 0.63 \mathrm{mmol})$ in $\mathrm{MeOH}(30 \mathrm{~mL})$ at ambient temperature. The suspension was stirred for $5 \mathrm{~h}$ and then concentrated, and the resulting solution was extracted $\left(\mathrm{CH}_{2} \mathrm{Cl}_{2}\right)$. The organic phase was dried $\left(\mathrm{Na}_{2} \mathrm{SO}_{4}\right)$, and volatiles were evaporated. The residue was chromatographed to give $4(0.46 \mathrm{~g}$, 89\%) as a solid foam: ${ }^{1} \mathrm{H}$ NMR $\delta 0.93(\mathrm{~d}, J=6.4 \mathrm{~Hz}, 6 \mathrm{H}), 1.73-1.77(\mathrm{~m}, 3 \mathrm{H}), 2.08,2.18(2 \times \mathrm{s}$, $2 \times 6 \mathrm{H}), 2.26(\mathrm{~s}, 9 \mathrm{H}), 2.28,2.31(2 \times \mathrm{s}, 2 \times 3 \mathrm{H}), 3.65-3.69(\mathrm{~m}, 2 \mathrm{H}), 4.72-4.83(\mathrm{~m}, 3 \mathrm{H}), 6.11(\mathrm{t}, J$ $=5.4 \mathrm{~Hz}, 1 \mathrm{H}), 6.33(\mathrm{t}, J=5.2 \mathrm{~Hz}, 1 \mathrm{H}), 6.43(\mathrm{~d}, J=4.9 \mathrm{~Hz}, 1 \mathrm{H}), 6.79,6.83,6.86(3 \times \mathrm{s}, 3 \times 2 \mathrm{H})$,

8.41, 8.99 (2× s, $2 \times 1 \mathrm{H}) ;{ }^{13} \mathrm{C}$ NMR $\delta 20.1,20.2,21.36,21.42,22.3,27.7,30.4,52.0,63.3,71.4$, 74.0, 81.2, 87.8, 128.5, 128.9, 129.0, 129.1, 129.9, 130.4, 135.4, 136.0, 136.1, 140.2, 140.5, 140.7, 146.6, 152.3, 154.0, 154.5, 168.6, 168.8, 169.7; HRMS (FAB, p-nitrobenzyl alcohol) $\mathrm{m} / z$ $825.3533\left[\mathrm{MH}^{+}\left(\mathrm{C}_{45} \mathrm{H}_{54} \mathrm{~N}_{4} \mathrm{O}_{9} \mathrm{~S}\right)=825.3535\right]$.

\section{6-(4-Methoxyphenyl)-9-[2,3,5-tri- $O$-(2,4,6-trimethylbenzoyl)- $\beta$-D-}

ribofuranosyl]purine (1c). (Procedure B). Treatment of $4(50 \mathrm{mg}, 0.061 \mathrm{mmol})$ and 4methoxyphenylboronic acid (18 $\mathrm{mg}, 0.12 \mathrm{mmol})$ by a modification of general method 2 [dried THF was used as solvent (rather than toluene) and heating for $8 \mathrm{~h}$ was at $60{ }^{\circ} \mathrm{C}$ (rather than 90 $\left.{ }^{\circ} \mathrm{C}\right)$ ] gave 1c (39 mg, 81\%) as a solid glass with ${ }^{1} \mathrm{H}$ and ${ }^{13} \mathrm{C}$ NMR spectra identical to those of 1c from general method 2 (procedure A).

(1) Kawakami, H.; Matsushita, H.; Naoi, Y.; Itoh, K.; Yoshikoshi, H. Chem. Lett. 1989, 235-238.

(2) Liu, J.; Robins, M. J. Org. Lett. 2004, 6, 3421-3423.

(3) Hocek, M.; Holy, A.; Votruba, I.; Dvorakova, H. Collect. Czech. Chem. Commun. 2000, 65, $1683-1697$.

(4) Liu, J.; Janeba, Z.; Robins, M. J. Org. Lett. 2004, 6, 2917-2919.

(5) Hocek, M.; Holy, A; Votruba, I.; Dvorakova, H. J. Med. Chem. 2000, 43, 1817-1825. 